\title{
Pathological findings in cornea tissue of patients with penetrating keratoplasty
}

\author{
Helga Kostelná1, Ján Rosocha1, Edita Paulíková2, Igor Kozák³, Mária Antalová2, \\ Martin Mahel ${ }^{4}$, Denisa Harvanová1, Jozefína Petrovičová ${ }^{5}$, Ladislav Bobák ${ }^{1}$
}

\author{
${ }^{1}$ Associated Tissue Bank of Faculty of Medicine, P. J. Šafárik University and L. Pasteur Faculty Hospital, \\ Trieda SNP 1, Košice, Slovakia \\ ${ }^{2}$ Institute of Biology and Ecology, Faculty of Science, P. J. Šafárik University, Košice, Slovakia \\ ${ }^{3}$ University of Calfornia San Diego, Shiley Eye Center, U.S.A. \\ ${ }^{4}$ Department of Ophthalmology, L. Pasteur Faculty Hospital, Košice, Slovakia \\ ${ }^{5}$ Institute of Medical Informatics, Faculty of Medicine, P. J. Safárik University, Slovakia
}

\begin{abstract}
The aim of this clinical study was to examine corneas extracted from the patients during penetrating keratoplasty for the presence of immunoglobulins and inflammatory cells that can be warning for the graft failure. Individual clinical diagnoses were correlated to the presence of the inflammatory signs in corneal tissue. The signs of inflammation in corneal layers were detected especially in group of patients classified as viral keratitis but were also found in corneas of patients with degenerative diseases of the cornea. Depending on the number of keratoplasties no statistical difference in analysed parameters was found. Inflammatory process represented by slight positive presence cellular infiltration and/or immunoglobulins could be present in corneal tissue also in absence of acute manifestation. Histological and immunohistochemical evaluation of corneal tissue from patients undergoing penetrating keratoplasty could improve estimation of correct diagnosis. Subsequently prompt adequate therapy could improve worse prognosis of corneal graft with evident immune process.
\end{abstract}

Key words: human cornea, immunoglobulins, keratoplasty, leukocyte infiltration

\section{Introduction}

Corneal damage is one of leading causes of blindness. Keratoplasty is a surgical procedure in which a part or all of a damaged or diseased cornea is replaced by healthy donor corneal tissue. The major goals of corneal transplantation are to improve the vision, reduce the pain and repair structural damage to maintain the integrity of the globe.

There are a number of risk factors that can help to predict the likelihood of corneal graft rejection. These include corneal vascularization, repeated keratoplasty [1-3], glaucoma [1,3,4], human leukocyte antigens [1] and blood type incompatibility [3], larger and eccentric grafts [1], host age [2], but also pre-operative ocular infection [4]. The corneal immune response to anti-

Correspondence: H. Kostelná, Associated Tissue Bank of Faculty of Medicine, P. J. Šafárik University and L. Pasteur Faculty Hospital, Trieda SNP 1, 04066 Košice, Slovakia; tel.: (+42155) 6404303, e-mail: helga.kostelna@upjs.sk gens results from an influx of cells from blood vessels of the limbus, iris/ciliary body, and neo-vascular elements in the cornea and diffusion of immunoglobulins, complement components and other active molecules into the cornea. Immunoglobulin $\mathrm{G}$ and immunoglobulin A were found in similar concentrations in the peripheral and central cornea. Immunoglobulin $\mathrm{M}$ was found in the periphery of the cornea probably because its larger molecular weight restricts its diffusion into the central cornea [5-7]. Inflamed surgical field increases the possibility of transplantation failure. Prevention lies in early detection of any signs of inflammatory process followed by appropriate therapy. Corticosteroid and immunosuppressive treatment is the therapy of choice [8-10].

The aim of this clinical study was to examine corneal buttons extracted from the patients undergoing penetrating keratoplasty for the presence of basic immunological inflammatory signs that can be warning for the failure of transplantation result. Cellular proinflammatory influx and immunoglobulins presence in 
Table 1. Number of patients in individual groups of diagnoses (number of corneas in parenthesis).

\begin{tabular}{|l|c|c|c|}
\hline \multicolumn{2}{|c|}{ Diagnosis } & \multicolumn{2}{c|}{$\mathrm{n}$} \\
\hline Keratoconus & 17 & $(20)$ \\
\hline \multicolumn{2}{|l|}{ Bullous keratopaty } & 65 & $69)$ \\
\hline \multirow{2}{*}{ Keratitis } & Viral & 7 & $(8)$ \\
\cline { 2 - 4 } & Microbial & 7 & $(8)$ \\
\hline \multirow{2}{*}{ Leucoma } & 8 & $(10)$ \\
\hline Other degenerative diseases and dystrophies & 12 & $(15)$ \\
\hline
\end{tabular}

$\mathrm{n}$ - number of patients (corneas)

corneal tissue were chosen for detection of immunological and/or inflammatory process in the cornea, which might influence the status of the cornea integrity and its homeostasis. Keratoconus, bullous keratopathy, keratitis, post-traumatic leucoma and other corneal degenerative diseases and dystrophies of the cornea were pre-operative clinical indications for penetrating keratoplasty. Immunoglobulins and cellular infiltration were evaluated in tissues extracted from diseased eyes as well as in corneas from healthy donors excluded from transplantation due to morphological reasons (such as stromal opacities, endothelial cell count less than $1500 / \mathrm{mm}^{2}$ ). The result of an evaluation can contribute to timely and correct treatment after cornea transplantation and thus to ensure the graft function and survival.

\section{Material and methods}

Corneal buttons. 130 corneal button specimens were acquired from 116 patients who underwent penetrating keratoplasty (6 years study). Analysed group consisted of 60 men and 56 women. The average age of patients was $61.42 \pm 16.75$ years, in the range of 12 to 97 years. Twenty corneas were obtained from 17 patients with keratoconus as primary indication for keratoplasty, 69 corneas from 65 patients with bullous keratopathy, 16 corneas from 14 patients with keratitis (classified as non-viral -8 from 7 patients, bacterial and fungal -8 from 7 patients), 10 corneas from 8 patients with leucoma after chemical, thermal or physical injury and 15 corneas from 12 patients with other degenerative diseases and dystrophies of the cornea (Table 1). All patients suffered from chronic disease persisting more than half a year. Patients were treated only with antibiotics and corticosteroids administrated locally. Immunosuppressive therapy was not applied in any of them before transplantation, so the corneal tissue could not be affected. The patients underwent the first penetrating keratoplasty in 78 cases, repeated keratoplasty in the same eye in 36 cases and repeated keratoplasty in the opposite eye in 16 cases.

The corneas of 6 cadaver donors were used as a control group. There were 3 men and 3 women in control group; the average age of the donors was $55.3 \pm 7.84$ years (in the range of 48 to 68 years). The donor corneas were considered not being suitable for surgical purposes after evaluation.

Processing of corneal buttons. All corneas (donor and patient) were fixed by $10 \%$ formaldehyde, patient corneas immediately after the excision and donor corneas 2-6 hours after procurement. Tissue was then paraffin embedded and cut into $4 \mu \mathrm{m}$ thick sections. One slide from each patient and donor sample was stained with haematoxylin and eosin dye and examined for leukocyte infiltration using the light microscope Alphaphot-2, type 119 (Nikon, Japan) at 200 up to $1000 \times$ magnifications. Other slides were stored at room temperature until they were immunohistochemically examined for the presence of immunoglobulins.

Investigation of immunoglobulins in corneal buttons. The slides were defatted 2 times in xylene and 3 times in methanol for 5 minutes in order to remove the paraffin. They were then rehydrated in phosphate saline-buffered solution (PBS), ( $\mathrm{pH}$ 7.2-7.4) for 10 minutes. Horseradish peroxidase conjugated swine anti-human immunoglobulins (SwAHu/Ig-Px) (Sevapharma, Prague, Czech Republic) was applied on the slides for 30 minutes. After 3 washings for 10 minutes in PBS the slides were incubated in $0.077 \%$ (w/v) 3,3,-diaminobenzidine tetrahydrochlorid dehydrate (DAB) and $0.075 \%(\mathrm{v} / \mathrm{v})$ hydrogen peroxide in PBS for 15 minutes. The slides were then washed 2 times in PBS and 1 time in distilled water for 10 minutes and dried. They were examined under the light microscope. The color intensity of the corneal layers in patients was compared with that of control group. It was evaluated as negative, slight positive or strong positive. To avoid interobserver variability caused by subjective mistakes all slides were evaluated by the same person in a blind fashion.

Ethical issues. This prospective clinical study was approved by ethic committee of the Faculty of Medicine of P. J. Safarik University.

Statistical analysis. The percentage of corneas with positive findings of cellular infiltration and immunoglobulins in epithelium and stroma in individual diagnosis groups and in all patients was calculated. The same was done with corneas in patients after the first keratoplasty, repeated keratoplasty and keratoplasty in the opposite eye. Separate groups were compared and the results were statistically processed and evaluated using software MS Excel 2000 and statistical programs Arcus Quickstat Biomedical 1.1. Yates' corrected chi-square test and/or Fisher exact test were used for the analysis.

\section{Results}

Light microscopy of corneal buttons showed normal corneal tissue in donors (Fig. 1a). In patients, only two of three basic corneal layers were noticed (epithelium and stroma). In some samples single endothelial cells could be seen. Most likely this was due to the patients' diagnoses and/or processing.

\section{Cellular infiltration}

The slides stained with haematoxylin and eosin dye were examined for the presence of inflammatory cells. Inflammatory cells were massively present in all clinically determined diagnoses (Table 2). Massive leukocyte infiltration (Fig. 1b) was found in 31 corneas of all samples $(23.8 \%)$. Inflammatory cells were found especially in the stroma, occasionally in the subepithelium. Polymorphonuclear neutrophilic leukocytes were dominant population in 28 corneas. In other 

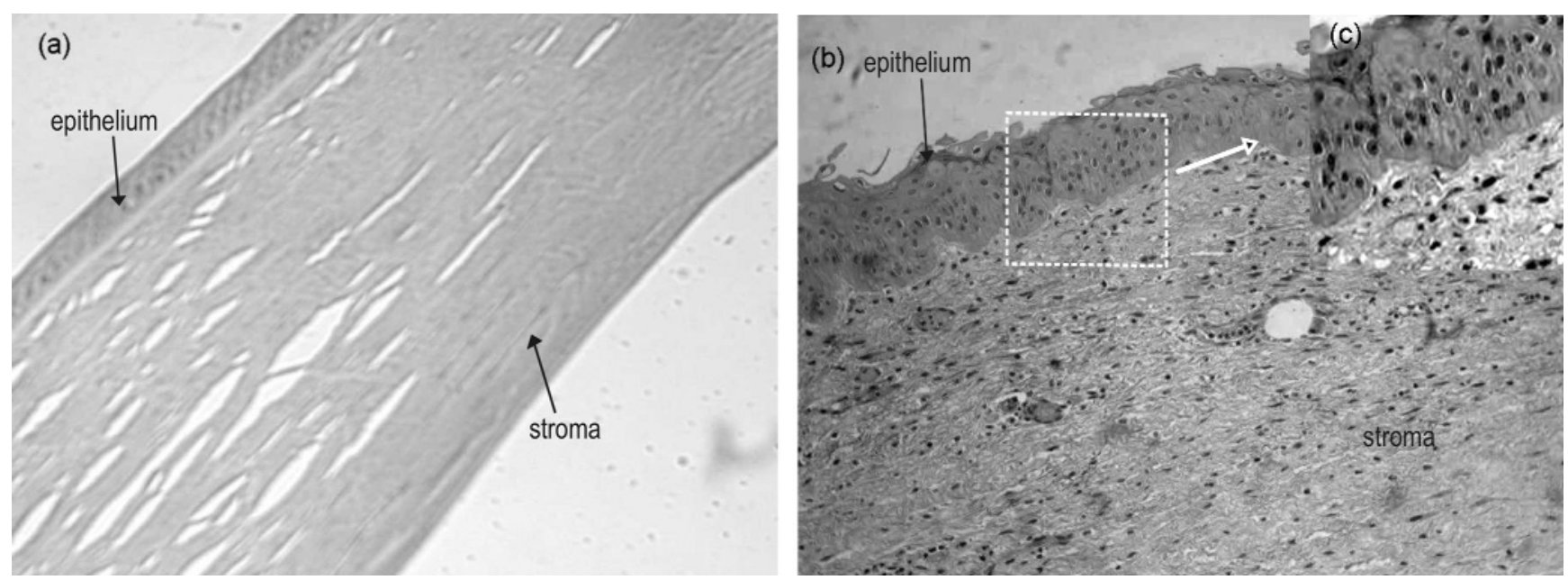

Fig. 1. Histological sections of human cornea evaluated by light microscopy. (a) Donor - normal structure of epithelium and stroma, (b, c) patient with clinical diagnosis keratitis - arrow indicates multiplayer epithelium many polymorphonuclear leukocytes can be seen in stroma. Stained with haematoxylin and eosin; $(\mathbf{a}, \mathbf{b})$ original magnification $\times 150$, inset $(\mathbf{c})$ original magnification $\times 400$.

3 corneas mononuclear cells were the main population of cellular infiltrate but they were present simultaneously with neutrophils. The presence of inflammatory cells was most frequent in patients with viral keratitis $(62.6 \%)$, post-traumatic leucoma $(60.0 \%)$ and other degenerative diseases of the cornea and dystrophies $(46.7 \%)$. The frequency of leukocytes cellular infiltration in patients with these three diagnoses was statistically significant when compared with those of bullous keratopathy $(p=0.002$ for viral keratitis, $p=0.001$ for leucoma and $p=0.002$ for other degenerative diseases and dystrophies of the cornea). Moreover, in 9 corneas $(6.9 \%)$ with positive cellular infiltration, the vascularization of the cornea in histological section was observed. There was no cellular inflammatory infiltration or vascularization in donor corneas.

\section{Presence of immunoglobulins}

The samples stained with SwAHu/Ig-Px and DAB were examined for the presence of immunoglobulins under light microscope. In all donors the intensity of the color (the presence of immunoglobulins) of the epithelium and stroma was very weak (Fig. 2a). Only patients' corneas with strong positive immunoglobulins were subsequently statistically analyzed. Immunoglobulins were noticeable in 17 cases $(13.1 \%)$ of all (Table 2). In 13 corneas (10.0\%) they were located in the epithelium (Fig. 2b) and in 8 corneas (6.2\%) in the stroma (Fig. 2c). In 4 corneas immunoglobulins were present in the epithelium and in the stroma as well (Fig. 2d). The most frequent presence of immunoglobulins in the epithelium was in the group of patients with viral keratitis $(37.5 \%$ of patients with this clinical diagnosis). The same group of patients was also the most often positive for the presence of immunoglobulins in the stroma $(25.0 \%$ positive corneas). However, there was no statistically significant difference in immunoglobulins presence when compared the group of patients suffering from viral keratitis with other diagnosis groups.

Some corneas were positive for only one parameter investigated. In other corneas cellular infiltration as well as immunoglobulins and/or vascularization were present simultaneously (Table 3 ).

\section{Pathological findings and repeated keratoplasty}

36 corneas of all 130 were acquired from patients that underwent repeated keratoplasty and 16 corneas from patients with the second keratoplasty but in the opposite eye. The most positive findings (in relative numbers) of cellular infiltration, immunoglobulins in epithelium and immunoglobulins in stroma were in corneas obtained from patients with repeated keratoplasty (Table 4 ) $-36.1 \%, 13.9 \%$ and $8.3 \%$ respectively. Statistical analysis did not confirm the difference in presence of any pathological finding in the corneas dependent on the number of keratoplasties.

\section{Discussion}

The regulation of immune response in the eye is complex and involves both soluble and cellular factors. This study was performed on 130 corneal buttons acquired from patients with various primary pre-operative clinical indications for cornea transplantation. During evaluation of histological specimens massive cellular infiltration was discovered in almost one quarter of corneas. It was found especially in patients with typical inflammatory diseases (keratitis of viral or other infectious origin) but was also present in patients with diagnoses that usually 
Table 2. Absolute numbers and percentage of corneas with strong positive findings of cellular infiltration, vascularization, and immunoglobulins in epithelium and stroma in individual diagnosis groups and in all corneas.

\begin{tabular}{|l|c|c|c|c|c|c|c|}
\hline & \multirow{2}{*}{ Keratoconus } & \multirow{2}{*}{$\begin{array}{c}\text { Bullous } \\
\text { keratopathy }\end{array}$} & Viral & $\begin{array}{c}\text { Microbial } \\
\text { origin }\end{array}$ & $\begin{array}{c}\text { Post-traumatic } \\
\text { leucoma }\end{array}$ & $\begin{array}{c}\text { Other } \\
\text { degenerative } \\
\text { disease and } \\
\text { dystrophies }\end{array}$ & \begin{tabular}{c} 
All corneas \\
\cline { 5 - 7 }
\end{tabular} \\
\cline { 6 - 8 } & $\mathrm{n}=20$ & $\mathrm{n}=69$ & $\mathrm{n}=8$ & $\mathrm{n}=8$ & $\mathrm{n}=10$ & $\mathrm{n}=15$ & $\mathrm{n}=130$ \\
\hline $\begin{array}{l}\text { Cellular } \\
\text { infiltration }\end{array}$ & $3(15.0 \%)$ & $7(10.2 \%)$ & $5(62.6 \%)$ & $3(37.5 \%)$ & $6(60.0 \%)$ & $7(46.7 \%)$ & $31(23.8 \%)$ \\
\hline Vascularization & $1(5.0 \%)$ & $3(4.3 \%)$ & $0(0.0 \%)$ & $0(0.0 \%)$ & $1(10.0 \%)$ & $4(26.7 \%)$ & $9(6.9 \%)$ \\
\hline Ig in epithelium & $3(15.0 \%)$ & $4(5.8 \%)$ & $3(37.5 \%)$ & $0(0.0 \%)$ & $2(20.0 \%)$ & $1(6.7 \%)$ & $13(10.0 \%)$ \\
\hline Ig in stroma & $0(0.0 \%)$ & $4(5.8 \%)$ & $2(25.0 \%)$ & $0(0.0 \%)$ & $1(10.0 \%)$ & $1(6.7 \%)$ & $8(6.2 \%)$ \\
\hline $\begin{array}{l}\text { Ig in epithelium } \\
\text { and/or stroma }\end{array}$ & $3(15.0 \%)$ & $6(8.7 \%)$ & $4(50.0 \%)$ & $0(0.0 \%)$ & $2(20.0 \%)$ & $1(6.7 \%)$ & $16(12.3 \%)$ \\
\hline
\end{tabular}

Ig - immunoglobulins; $\mathrm{n}$ - number of corneas
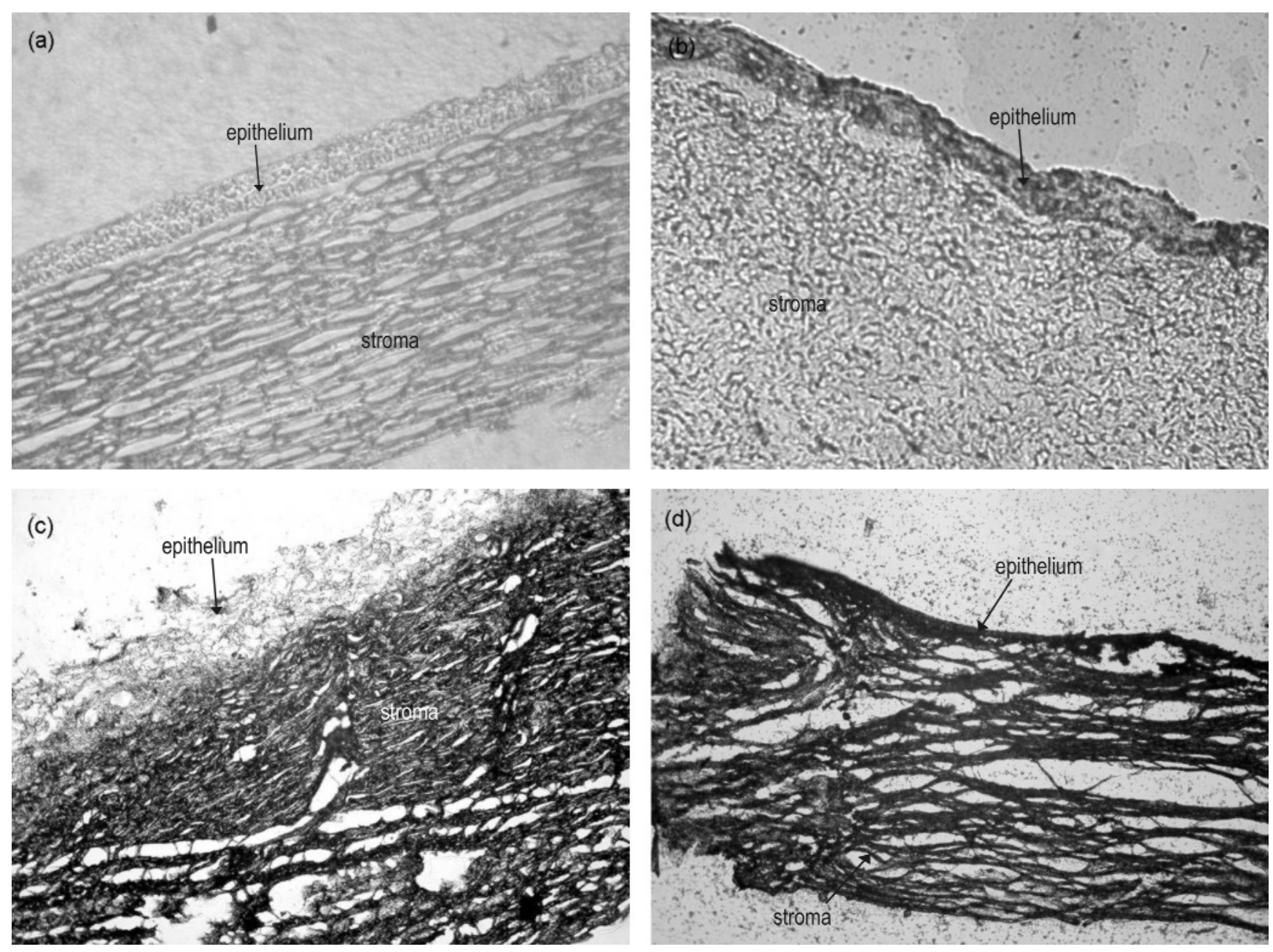

Fig. 2. Human corneas examined by light microscopy for the presence of immunoglobulins using SwAHu/Ig-Px staining. (a) Donor weak intensity of colour staining of epithelium as well as stroma (negative result), (b) patient with post-traumatic leucoma - positive immunohistochemical staining of immunoglobulins in epithelium, (c) patient with bullous keratopathy - positive staining of immunoglobulins in stroma, (d) patient with bullous keratopathy - positive staining of immunoglobulins in epithelium and stroma. (a, d) original magnification $\times 120,(\mathbf{b}, \mathbf{c})$ original magnification $\times 150$. 
Table 3. Analysis of 41 corneas $(100.0 \%)$ with risk factors for graft failure - numbers of corneas.

\begin{tabular}{|l|c|}
\hline \multicolumn{1}{|c|}{ Risk factor } & $\begin{array}{c}\text { Number of } \\
\text { corneas }\end{array}$ \\
\hline Only cellular infiltration & $16(39.0 \%)$ \\
\hline Cellular infiltration and vascularization & $8(19.5 \%)$ \\
\hline Only immunoglobulins & $10(24.4 \%)$ \\
\hline Cellular infiltration and immunoglobulins & $6(14.6 \%)$ \\
\hline $\begin{array}{l}\text { Cellular infiltration and immunoglobulins and } \\
\text { vascularization }\end{array}$ & $1(2.4 \%)$ \\
\hline
\end{tabular}

are not associated with an inflammatory process (e.g. keratoconus, bullous keratopathy and other degenerative diseases of the cornea). In keratoconus predisposition of the cornea to the infection could be due to contact lens wearing [11]. According Lema et al. [12] increased levels of interleukin-6, TNF- $\alpha$, and MMP-9 in tears of patients with keratoconus indicate that the pathogenesis of keratoconus may involve also chronic inflammatory events. In bullous keratopathy swelling of the epithelium can lead to the rupture of bullae. Subsequent epithelial defects can induce infection and inflammation in the cornea. The occurrence of bullae and prolonged time of the disease are risk factors for infection in bullous keratopathy [13]. Detection of corneal cellular infiltration in patients with different types of corneal degenerative diseases in our study is in agreement with these findings. High occurrence of inflammatory cellular infiltration in post-traumatic leukomas showed that the tissue was in stage of inflammation and not regeneration in time of transplantation.

The occurrence of cellular infiltration in patients with repeated keratoplasty was higher $(36.1 \%)$ than in patients undergoing the first one $(20.5 \%)$, Table 4. However, the difference was not statistically significant. The reason of graft failure may not lie only in immunologically related rejection but also in physical and biomechanical properties of the donor cornea and clinical condition of the patient [1-4].

Corneal trauma can encourage new blood vessels to grow from the limbus. Neovascularisation is generally accompanied with an inflammatory response. In our study 9 corneas of those with positive cellular infiltration were positive for vascularisation as well. Vascularisation was present not only in corneas before the first transplantation (4 cases) but also in transplanted corneas ( 5 cases).

Immunoglobulins present in patients' corneas could be the result of immune response to infectious agents, corneal antigens and (in repeated keratoplasties) alloantigens. Local production of antibodies specific to different viral or microbial agents in cornea has been documented [14-16]. In our study some corneas were positive for immunoglobulins and inflammatory cells
Table 4. Absolute numbers and percentage of corneas positive for the presence of cellular infiltration, immunoglobulins in epithelium and stroma depending on the number of keratoplasties

\begin{tabular}{|l|c|c|c|}
\hline & $\begin{array}{c}\text { First } \\
\text { keratoplasty }\end{array}$ & $\begin{array}{c}\text { Repeated } \\
\text { keratoplasty }\end{array}$ & $\begin{array}{c}\text { Keratoplasty } \\
\text { in the } \\
\text { opposite eye }\end{array}$ \\
\cline { 2 - 4 } & $\mathrm{n}=78$ & $\mathrm{n}=36$ & $\mathrm{n}=16$ \\
\hline $\begin{array}{l}\text { Cellular } \\
\text { infiltration }\end{array}$ & $16(20.5 \%)$ & $13(36.1 \%)$ & $2(12.5 \%)$ \\
\hline $\begin{array}{l}\text { Ig in } \\
\text { epithelium }\end{array}$ & $6(7.7 \%)$ & $5(13.9 \%)$ & $2(12.5 \%)$ \\
\hline Ig in stroma & $5(6.4 \%)$ & $3(8.3 \%)$ & $0(0.0 \%)$ \\
\hline
\end{tabular}

simultaneously. We suppose it could be the result of an infectious inflammatory process. Ten corneas were positive for the presence of immunoglobulins and without cellular infiltration. Six of them were from patients that underwent the repeated keratoplasty. We can think about alloantibodies in mentioned patients. To distinguish the exact character of these antibodies further investigations are required.

There was higher occurrence of immunoglobulins in epithelium (13 corneas) than in stroma (8 corneas) in analysed group. It could be due to the fact that Langerhans antigen presenting cells are present especially in the epithelium.

In many others analysed corneas (the results of which are not included in the present study) weak infiltration of inflammatory cells and weak positive immunohistochemical staining of immunoglobulins were frequently observed. Also these results point to inflammatory process present in corneal tissue. We suppose that an inflammation may be present also in absence of acute manifestation.

Implantation of donor cornea with recipient's tissue without evident signs of inflammation would complicate the process of grafting. In these cases another approach of patient's treatment after cornea transplantation would be suitable. In conclusion, we recommend simple and prompt histological and/or immunohistochemical examination of corneal tissue from the patients after penetrating keratoplasty. The results may serve for better determination of pre-transplant diagnosis and post-keratoplasty treatment of patients.

Acknowledgements: We would like to acknowledge the Institute of Ophthalmology, Faculty of Medicine, P. J. Šafárik University and L. Pasteur Faculty Hospital Košice and the Department of Ophthalmology of Saint Cyril and Method Hospital Bratislava for cooperation in this clinical study.

\section{References}

[ 1] Boisjoly HM, Tourigny R, Bazin R, Laughrea PA, Dube I, Chamberland G, Bernier R, Roy R. Risk factors of corneal graft failure. Ophthalmology. 1993;100(11):1728-1735. 
[2] Inoue K., Amano S., Oshika T., Tsuru T. Risk factors for corneal graft failure and rejection in penetrating keratoplasty. Acta Ophthalmol Scand. 2001;79(3):251-255.

[3] Maguire MG, Stark WJ, Gottsch JD, Stulting RD, Suguar A, Fink NE, Schwartz A. Risk factors for corneal graft failure and rejection in the collaborative corneal transplantation studies. Collaborative Corneal Transplantation Studies Research Group. Ophthalmology. 1994;101(9):1536-1547.

[4] Wilson SE, Kaufman HE. Graft failure after penetrating keratoplasty. Surv Ophthalmol. 1990;34(5):325-356.

[5] Verhagen C, Breebaart AC, Kijlstra A. Diffusion of immunoglobulin $\mathrm{G}$ from the vascular compartment into the normal rabbit cornea. Invest Ophthalmol Vis Sci. 1990; 31(8):1519-1525.

[ 6] Van Der Veen G, Broersma L, Bruyne I, Verhagen C, Ruijter J, Van Rij G, Van Der Gaag R. Influx of immunoglobulins from the vacsular compartment into a grafted cornea. Invest Ophthalmol Vis Sci; 38(5):876-883.

[7] Pleyer U, Bergmann L, Krause A, Hartmann C. Autoimmune diseases of the peripheral cornea. Immunopathology, clinical aspects and therapy. Klin Monatsbl Augenheilkd. 1996;208: 73-81.

[ 8] Hill JC. The use of systemic cyclosporin in a human corneal transplantation: a preliminary report. Doc Ophthalmol. 1986; 62:337-344.

[9] Hill JC, Ivey A. Corticosteroids in corneal graft rejection: double vs single pulse therapy. Cornea. 1994;13(5):383-388.
[10] Benelli U, Lepri A, Del Tacca M, Nardi M. FK-506 delays corneal graft rejection in a model of corneal xenotransplantation. J Ocul Pharmacol Ther. 1996;12:425-431.

[11] Junko E, Tooru S, Yumi K, Jun S, Mitsuru S. Two cases of keratoconus developed bacterial infection masquerading acute hydrops. Ophthalmology. 2000;42(5):711-714.

[12] Lema I, Durán JA. Inflammatory molecules in the tears of patients with keratoconus. Ophthalmology. 2005;112(4):654659.

[13] Luchs JI, Cohen EJ, Rapuano CJ, Laibson PR. Ulcerative keratitis in bullous keratopathy. Ophthalmology. 1997;104(5): 816-822.

[14] Thakur A, Kyd J, Xue M, Willcox MDP, Cripps A. Effector mechanisms of protection against Pseudomonas aeruginosa keratitis in immunized rats. Infect Immun. 2001;69:32953304.

[15] Robert PY, Liekfeld A, Metzner S, Ranger-Rogez S, Adenis JP, Denis F, Hartmann Ch, Pleyer U. Specific antibody production in herpes keratitis: intraocular inflammation and corneal neovascularisation as predicting factors. Graefe's Arch Clin Exp Ophthalmol. 2006 244:210-215.

[16] Zhang H, Chen H, Niu J, Wang Y, Xie L. Role of adaptive immunity in the pathogenesis of Candida albicans keratitis. Invest Ophthalmol Vis Sci. 2009;50:2653-2659.

Submitted: 25 September, 2009 Accepted after reviews: 11 February, 2010 